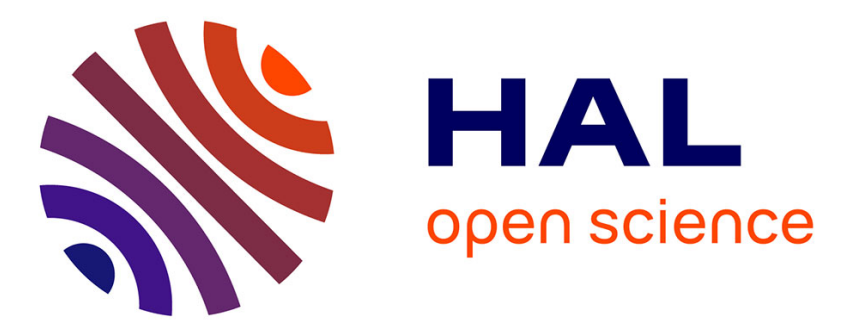

\title{
Capital-labor Substitution and Endogenous Fluctuations: a Monopolistic Competition Approach with Variable Mark-up
}

Thomas Seegmuller

\section{- To cite this version:}

Thomas Seegmuller. Capital-labor Substitution and Endogenous Fluctuations: a Monopolistic Competition Approach with Variable Mark-up. Japanese Economic Review, 2009, 60 (3), pp.301-319. 10.1111/j.1468-5876.2008.00452.x . halshs-00194292

\section{HAL Id: halshs-00194292 \\ https://shs.hal.science/halshs-00194292}

Submitted on 6 Dec 2007

HAL is a multi-disciplinary open access archive for the deposit and dissemination of scientific research documents, whether they are published or not. The documents may come from teaching and research institutions in France or abroad, or from public or private research centers.
L'archive ouverte pluridisciplinaire HAL, est destinée au dépôt et à la diffusion de documents scientifiques de niveau recherche, publiés ou non, émanant des établissements d'enseignement et de recherche français ou étrangers, des laboratoires publics ou privés. 


\title{
Capital-labor Substitution and Endogenous Fluctuations: a Monopolistic Competition Approach with Variable Mark-up
}

\author{
Thomas Seegmuller*
}

November 14, 2007

\begin{abstract}
In macroeconomics, economists introduce most frequently imperfect competition on product markets using the Dixit and Stiglitz (1977) monopolistic competition model. However, by assumption, this framework ignores one important feature of imperfect competition: strategic interactions between producers. Taking into account this remark and following Yang and Hejdra (1993), this paper analyzes an overlapping generations model where strategic interactions between producers are introduced and examines how they affect the stability properties of the steady state. Because of free entry, strategic interactions between producers imply a new dynamic feature, mark-up variability, promoting indeterminacy and endogenous cycles. Indeed, in contrast to the model without strategic interaction, endogenous fluctuations can occur when the substitution between the production factors, capital and labor, is not too weak, but in accordance with empirical estimates.
\end{abstract}

JEL classification: D43, E32.

Keywords: Endogenous fluctuations, imperfect competition, strategic interactions, mark-up variability, capital-labor substitution, overlapping generations.

${ }^{*}$ CNRS, Paris School of Economics, University Paris 1, Centre d'Economie de la Sorbonne, 106-112 Boulevard de l'Hôpital 75647 Paris Cedex 13, France, Tel: (33) 14407 81 99, Fax: (33) 1440782 31, e-mail: seegmu@univ-paris1.fr. I would like to thank an anonymous referee for useful comments. I also thank Antoine d'Autume, Rodolphe Dos Santos Ferreira, Teresa Lloyd-Braga and Alain Venditti for valuable suggestions, and participants of seminars and conferences where this paper was presented. All remaining errors are mine. 


\section{Introduction}

The purpose of this paper is to study the role of strategic interactions between producers on the emergence of expectation driven fluctuations. We will show that taking into account the strategic behavior of firms drastically affects the dynamics, by promoting the emergence of endogenous fluctuations.

As it is well known, the most commonly used imperfectly competitive framework in macroeconomics is based on Dixit and Stiglitz (1977). In this monopolistic competition model, each firm acts as a monopolist on its own differentiated market, but has no influence on the behaviors of its competitors. As a corollary, each producer takes as given aggregate variables. Therefore, even if strategic interactions are a key element addressed by the introduction of imperfect competition, they are ignored, by assumption, in the Dixit and Stiglitz (1977) framework.

In this paper, we are still concerned with a monopolistic competition model with differentiated final goods, where the product diversity is assumed to be endogenously determined by a zero profit condition. However, following Yang and Hejdra (1993), strategic interactions are introduced assuming that firms take into account their influence on the aggregate price. Therefore, the optimal behavior of firms corresponds to a (differentiated) Nash equilibrium in prices. At equilibrium, the mark-up factor is not constant, as in the usual Dixit and Stiglitz (1977) framework, but is decreasing in the product diversity. Because of the free entry condition, the business formation (product diversity) is procyclical and, as a consequence, the mark-up factor is countercyclical. ${ }^{1}$ Note that these two dynamic properties are not simple curiosities but have a large empirical support (Bils (1987), Rotemberg and Woodford (1991), Portier (1995), Martins and Scarpetta (1999)).

To be able to compare our results with the usual monopolistic competition economy without strategic interaction, our model admits as a particular case the Dixit and Stiglitz (1977) framework where producers have no influence on the aggregate variables. Note that, in this configuration, the mark-up is constant.

To analyze the role of strategic interactions between producers on local indeterminacy and the occurrence of endogenous cycles, we introduce this imperfectly competitive model in a simple two-period lived overlapping gen-

\footnotetext{
${ }^{1}$ Note that, in a dynamic general equilibrium model, Gali (1995) also studies a monopolistic competition model where the mark-up factor depends on the product diversity. However, in contrast to our paper, his approach is not based on the strategic behavior of producers. Considering a monopolistic competition without strategic interaction, he rather assumes that the elasticity of substitution between the different varieties of goods is increasing in the product diversity, which is the source of mark-up variability.
} 
erations economy, where households consume in both periods, save through capital, and elastically supply labor at the first period of their life. ${ }^{2}$ To underline the key influence of the strategic behavior of firms on the dynamics, we first consider the usual monopolistic competition case without strategic interaction. We show that the results are similar to those obtained under perfect competition. Indeterminacy and endogenous cycles require a sufficiently small capital-labor substitution. In the particular case where households only consume at the second period of their life, we recover the results obtained by Reichlin (1986), i.e., indeterminacy and cycles require an elasticity of capitallabor substitution smaller than the capital share in income, that needs to be smaller than one half.

On the contrary, under strategic interactions and mark-up variability, endogenous fluctuations emerge when capital and labor are high substitutes. Indeterminacy occurs for a large range of elasticities of capital-labor substitution, that encompasses the Cobb-Douglas case and all values in accordance with empirical studies (see Duffy and Papageorgiou (2000)). ${ }^{3}$ It is also interesting to notice that indeterminacy does not require large increasing returns, as emphasized by recent empirical results (Basu and Fernald (1995), Burnside (1996), Basu and Fernald (1997)). ${ }^{4}$ Furthermore, the limit case often considered in the literature where households do not consume at the first period $^{5}$ seems to be quite specific. Indeed, as soon as households have positive consumptions in both period, a higher degree of mark-up variability reduces the range of capital-labor substitution for indeterminacy.

More generally, since the model developed in this paper shows that strategic interactions between producers and mark-up variability promote the occurrence of endogenous fluctuations, this underlines that imperfect competition on the product market is still an important channel to understand expectation driven fluctuations, as soon as a sufficiently departure from the perfect competition is considered.

This paper is organized as follows. In Section 2, we present the model. In Section 3, we establish the existence of a steady state. We study local dynamics in Section 4 and conclude in Section 5. Several proofs are given in

\footnotetext{
${ }^{2}$ In overlapping generations economies, the relationship between endogenous fluctuations and mark-up variability due to business formation has also been studied by Kuhry (2001), Seegmuller (2003) and Dos Santos Ferreira and Lloyd-Braga (2005). However, in contrast to our analysis, all these papers assume the product diversity as fixed.

${ }^{3}$ See especially the numerical illustration at the end of Section 4.

${ }^{4}$ See Cazzavillan (2001) or Lloyd-Braga, Nourry, and Venditti (2007) for closely related results obtained in overlapping generations models with productive externalities.

${ }^{5}$ See for instance Reichlin (1986), Cazzavillan (2001) or Dos Santos Ferreira and LloydBraga (2005).
} 
the Appendix.

\section{The Model}

\subsection{Consumers}

We consider an overlapping generations economy with perfect foresight and discrete time $t=1,2, \ldots,+\infty$. Population is constant and, for simplicity, we assume that the population size of each generation is normalized to one. Therefore, at each period, a representative consumer is born, living for two periods. He supplies labor $L_{t}$ at the first period of his life, consumes all the available goods at both period, and saves through the purchase of aggregate capital $K_{t}$ that firms use for the production in the next period. ${ }^{6}$ Household's preferences, defined over consumption in both period and leisure, are summarized by the following utility function:

$$
(1 / B) U\left(C_{1 t} / D, C_{2 t+1}\right)-v\left(L_{t}\right)
$$

where $B>0$ and $D>0$ are two scaling parameters, and $C_{1 t}$ (respectively $C_{2 t+1}$ ), which represents consumption at the first (second) period of life, is a single composite good represented by the aggregate of varieties:

$$
C_{j h}=N_{h}^{-\frac{1}{\varepsilon-1}}\left[\sum_{i=1}^{N_{h}} c_{i j h}^{\frac{\varepsilon-1}{\varepsilon}}\right]^{\frac{\varepsilon}{\varepsilon-1}}, j=1,2, h=t, t+1,
$$

where $c_{i j h}$ denotes the consumption of a single variety of good and $\varepsilon>1$ the elasticity of substitution between the different varieties. Even if equation (2) means that there is no taste for variety, ${ }^{7}$ we assume that the representative household consumes all the available goods at each period. We further consider that the capital good is represented by the same aggregate of varieties. Moreover, the utility function (1) has the following properties:

Assumption 1 The function $U\left(x_{1}, x_{2}\right)$ is continuous for $x_{1} \geq 0$ and $x_{2} \geq 0$ and has continuous derivatives of every required order for $x_{1}>0$ and $x_{2}>$ 0. Moreover, $U\left(x_{1}, x_{2}\right)$ is increasing in $x_{1}$ and $x_{2}$, strongly quasi-concave, homogeneous of degree one, and such that the indifference curves do not cross the axes.

\footnotetext{
${ }^{6}$ This means that capital, that fully depreciates after one period of use, is predetermined.

${ }^{7}$ See Benassy (1996) for more details.
} 
The function $v(L)$ is continuous for $0 \leq L \leq \bar{L}$ and has continuous derivatives of every required order for $0<L<\bar{L}$, where the labor endowment $\bar{L}$ may be finite or infinite. Furthermore, we assume that $v^{\prime}(L)>0, v^{\prime \prime}(L) \geq$ $0, \lim _{L \rightarrow 0} v^{\prime}(L)=0$ and $\lim _{L \rightarrow \bar{L}} v^{\prime}(L)=+\infty$.

The household born at period $t$ maximizes his utility function (1) under the two following budget constraints:

$$
\begin{gathered}
p_{t} C_{1 t}+p_{t} K_{t}=w_{t} L_{t} \\
p_{t+1} C_{2 t+1}=r_{t+1} K_{t}
\end{gathered}
$$

where $w_{t}$ is the nominal wage, $r_{t+1}$ the future nominal interest rate and $p_{t}$ the price of the composite final good. If we note $\omega_{t} \equiv w_{t} / p_{t}$ the real wage and $\rho_{t+1} \equiv r_{t+1} / p_{t+1}$ the real interest rate, the optimal consumptions and savings are given by:

$$
\begin{gathered}
C_{1 t}=\alpha\left(D \rho_{t+1}\right) \omega_{t} L_{t} \\
C_{2 t+1}=\left(1-\alpha\left(D \rho_{t+1}\right)\right) \rho_{t+1} \omega_{t} L_{t} \\
K_{t}=\left(1-\alpha\left(D \rho_{t+1}\right)\right) \omega_{t} L_{t}
\end{gathered}
$$

where $\alpha\left(D \rho_{t+1}\right) \in(0,1)$ represents the propensity to consume when young and $1-\alpha\left(D \rho_{t+1}\right)$ the propensity to save or the saving rate. ${ }^{8}$ As explained in the Appendix, the elasticity of intertemporal substitution in consumption is given by:

$$
\eta\left(D \rho_{t+1}\right)=1-\frac{\alpha^{\prime}\left(D \rho_{t+1}\right) D \rho_{t+1}}{\alpha\left(D \rho_{t+1}\right)\left(1-\alpha\left(D \rho_{t+1}\right)\right)}
$$

We can notice that $\alpha$ is increasing when there is intertemporal complementarity $(\eta<1)$ and is decreasing when there is intertemporal substitutability $(\eta>1)$. We now define the following function: ${ }^{9}$

$$
U^{*}\left(D \rho_{t+1}\right) \equiv U\left(\alpha\left(D \rho_{t+1}\right),\left(1-\alpha\left(D \rho_{t+1}\right)\right) D \rho_{t+1}\right)
$$

where $U^{*^{\prime}}\left(D \rho_{t+1}\right) D \rho_{t+1} / U^{*}\left(D \rho_{t+1}\right)=1-\alpha\left(D \rho_{t+1}\right)$. Then, the labor supply is determined by:

$$
(1 /(B D)) U^{*}\left(D \rho_{t+1}\right) \omega_{t}=v^{\prime}\left(L_{t}\right)
$$

Note that, under Assumption 1, the labor supply is increasing in the real wage. Using $\epsilon_{v}(L) \equiv v^{\prime \prime}(L) L / v^{\prime}(L) \in(0,+\infty)$, the elasticity of labor supply with respect to the real wage is equal to $1 / \epsilon_{v}(L) \in(0,+\infty)$.

\footnotetext{
${ }^{8}$ The existence of the function $\alpha\left(D \rho_{t+1}\right)$ is established in the Appendix.

${ }^{9}$ More details are given in the Appendix.
} 


\section{$2.2 \quad$ Firms}

In the production sector, $N_{t} \geq 2$ firms are active at period $t$. Each one produces a single variety of output $i$ using the technology:

$$
y_{i t}=A\left(f\left(a_{i t}\right) l_{i t}-\phi\right)
$$

where $A>0$ is a scaling parameter, $a_{i t}=k_{i t-1} / l_{i t}$ the capital-labor ratio, $l_{i t}$ the labor used by firm $i$ and $\phi>0$ a fixed cost. We further assume:

Assumption 2 The intensive production function $f(a)$ is continuous for $a \geq 0$, positively valued and differentiable as many times as needed for $a>0$, with $f^{\prime}(a)>0$ and $f^{\prime \prime}(a)<0$.

Note that (11) means that the technology is characterized by a constant marginal cost, but returns to scale are increasing because of the existence of the fixed cost $\phi>0$.

Because competition is imperfect, each producer maximizes its profits, facing the demand:

$$
d_{i t}=\frac{1}{N_{t}}\left(\frac{p_{i t}}{p_{t}}\right)^{-\varepsilon} \frac{I_{t}}{p_{t}}
$$

where $I_{t}$ is the aggregate income, $p_{i t}$ the price of variety $i$ and $p_{t}$ the price average, defined by $p_{t}=\left[\left(1 / N_{t}\right) \sum_{i=1}^{N_{t}} p_{i t}^{1-\varepsilon}\right]^{1 /(1-\varepsilon)}$.

Firms take as given the aggregate income $I_{t}$. However, to be able to underline the role of strategic interactions on the dynamics, we consider two cases. On the one hand, without strategic interaction between producers, each firm neglects its effect on the average price $p_{t}$, which is therefore taken as given, as usually in the monopolistic competition model developed by Dixit and Stiglitz (1977). This implicitly means that each producer considers ex ante the product diversity $N_{t}$ as sufficiently large. We deduce that the elasticity of demand $\nu_{i t} \equiv-\left(\partial d_{i t} / \partial p_{i t}\right)\left(p_{i t} / d_{i t}\right)$ is constant and equal to $\varepsilon$. On the other hand, with strategic interactions between producers, firms take into account their influence on the average price $p_{t}$ because, following Yang and Hejdra (1993), they do not consider ex ante the product diversity $N_{t}$ as arbitrarily large. Then, firms behave as a differentiated oligopoly in prices, i.e., their optimal behavior corresponds to a Nash equilibrium in prices. In this case, the elasticity of demand is no more constant, but is equal to $\nu_{i t}=$ $\varepsilon+(1-\varepsilon) p_{i t}^{1-\varepsilon} / \sum_{i=1}^{N_{t}} p_{i t}^{1-\varepsilon}$. To be able to analyze and compare these two imperfectly competitive models using the same framework, we assume that the elasticity of demand is defined by $\nu_{i t}=\varepsilon+\tau(1-\varepsilon) p_{i t}^{1-\varepsilon} / \sum_{i=1}^{N_{t}} p_{i t}^{1-\varepsilon}$, with $\tau \in\{0,1\}$. 
Since firms are identical, we focus on the first order conditions of profit maximization at the symmetric equilibrium:

$$
\begin{aligned}
\rho_{t} & =\left(1-1 / \nu_{t}\right) A R\left(a_{t}\right), \text { with } R\left(a_{t}\right)=f^{\prime}\left(a_{t}\right) \\
\omega_{t} & =\left(1-1 / \nu_{t}\right) A W\left(a_{t}\right), \text { with } W\left(a_{t}\right)=f\left(a_{t}\right)-a_{t} f^{\prime}\left(a_{t}\right) \\
\nu_{t} & =\varepsilon\left(1-\tau / N_{t}\right)+\tau / N_{t}>1, \text { with } N_{t} \geq 2
\end{aligned}
$$

where $k_{i t-1}=k_{t-1}, l_{i t}=l_{t}, a_{t}=k_{t-1} / l_{t}$ and $p_{i t}=p_{t}$.

Note that the mark-up factor is given by $\nu_{t} /\left(\nu_{t}-1\right)$. Without strategic interactions, i.e., $\tau=0$, this mark-up factor is constant and equal to $\varepsilon /(\varepsilon-1)$. On the contrary, with strategic interactions between producers, i.e., $\tau=1$, the mark-up is variable. Indeed, because the elasticity of demand is increasing with the product diversity, the mark-up factor $\nu_{t} /\left(\nu_{t}-1\right)$ is decreasing in $N_{t} \cdot{ }^{10}$

Assuming free entry and exit, the product diversity, or equivalently the number of firms, is determined by the usual zero profit condition:

$$
\nu_{t}=f\left(a_{t}\right) l_{t} / \phi
$$

Because the production of an individual firm is given by $y_{t}=A\left(f\left(a_{t}\right) l_{t}-\right.$ $\phi)$, this free entry condition is equivalent to $y_{t}=A \phi\left(\nu_{t}-1\right)$. Hence, in the absence of strategic interaction, the production of an individual firm is constant, because $\nu_{t}=\varepsilon$. On the contrary, with strategic interactions, we get a more reasonable result. Since $\nu_{t}$ depends on $N_{t}$, the individual production $y_{t}$ is no more constant.

\subsection{Equilibrium}

Equilibrium on the capital market is ensured by $N_{t} k_{t-1}=K_{t-1}$ and equilibrium on the labor market by $N_{t} l_{t}=L_{t}$. Then, using equations (15) and (16), we obtain:

$$
N_{t}=\frac{f\left(a_{t}\right) K_{t-1}}{\varepsilon \phi a_{t}}+\tau \frac{\varepsilon-1}{\varepsilon}
$$

This equation can be rewritten $N_{t}=Y_{t} /[A \phi(\epsilon-1)]+\tau$, where $Y_{t}=$ $N_{t} y_{t}$ is the aggregate production. This means that the product diversity is procyclical whatever the value of $\tau .{ }^{11}$

\footnotetext{
${ }^{10}$ Note that Gali (1995) has also analyzed, in a dynamic setting, a monopolistic competition model where the mark-up factor depends on the number of firms. However, his approach is different from ours because it is not founded on the strategic behavior of producers. In his model, the usual assumptions of Dixit and Stiglitz (1977) apply, except that he further assumes that the elasticity between the different varieties of goods is increasing in the product diversity $N_{t}$, which is the key mechanism responsible for mark-up variability.

${ }^{11}$ Since $N_{t} \geq 2$, equation (17) implies that $f\left(a_{t}\right) K_{t-1} /\left(\phi a_{t}\right) \geq \varepsilon(2-\tau)+\tau$.
} 
Using equations (15) and (17), the inverse of the mark-up factor is also defined by:

$$
\begin{aligned}
1-\frac{1}{\nu_{t}} & =\frac{(\varepsilon-1)\left(N_{t}-\tau\right)}{\varepsilon N_{t}-\tau(\varepsilon-1)} \\
& =\frac{\varepsilon-1}{\varepsilon}\left(1-\tau \frac{\phi a_{t}}{f\left(a_{t}\right) K_{t-1}}\right) \equiv m\left(a_{t}, K_{t-1}\right)
\end{aligned}
$$

Without strategic interactions $(\tau=0)$, the mark-up factor is equal to $\epsilon /(\epsilon-1)$ and is constant, as usually in the Dixit and Stiglitz (1977) model. With strategic interactions $(\tau=1)$, since the product diversity $N_{t}$ is procyclical and $\nu_{t} /\left(\nu_{t}-1\right)$ is decreasing in $N_{t}$, the mark-up factor $1 / m\left(a_{t}, K_{t-1}\right)$ is variable and countercyclical. Note that several empirical studies provide an empirical support for procyclical business formation and countercyclical mark-ups (Bils (1987), Rotemberg and Woodford (1991), Portier (1995), Martins and Scarpetta (1999)).

Substituting (13), (14) and (18) into equations (7) and (10), we determine the two dynamic equations:

$$
\begin{gathered}
K_{t}=\left[1-\alpha\left(D m\left(a_{t+1}, K_{t}\right) A R\left(a_{t+1}\right)\right)\right] m\left(a_{t}, K_{t-1}\right) A W\left(a_{t}\right) K_{t-1} / a_{t} \\
(1 /(B D)) U^{*}\left[D m\left(a_{t+1}, K_{t}\right) A R\left(a_{t+1}\right)\right] m\left(a_{t}, K_{t-1}\right) A W\left(a_{t}\right)=v^{\prime}\left(K_{t-1} / a_{t}\right)
\end{gathered}
$$

Definition 1 An intertemporal equilibrium with perfect foresight is a sequence $\left(K_{t-1}, a_{t}\right) \in \mathbb{R}_{++}^{2}, t=1,2, \ldots, \infty$, such that (19) and (20) are satisfied, given $K_{0}>0$.

Equations (19) and (20) rule the dynamics of the model and define a two-dimensional dynamic system with one predetermined variable, aggregate capital $K_{t-1}$. Given $\left(K_{t-1}, a_{t}\right)$, we are able to determine $\left(K_{t}, a_{t+1}\right)$.

\section{Existence of a Steady State}

A steady state of the dynamic system (19)-(20) is a solution $\left(K_{t-1}=K, a_{t}=\right.$ a), for all $t$, such that:

$$
\begin{gathered}
{[1-\alpha(D m(a, K) A R(a))] A=\frac{a}{m(a, K) W(a)}} \\
(1 /(B D)) U^{*}[D m(a, K) A R(a)] m(a, K) A W(a)=v^{\prime}(K / a)
\end{gathered}
$$


Following Cazzavillan, Lloyd-Braga, and Pintus (1998) and Aloi, Dixon, and Lloyd-Braga (2000), we ensure the existence of a steady state, namely with $K=\bar{K}, a=\bar{a},{ }^{12}$ by choosing appropriate values of the three scaling parameters $A>0, B>0$, and $D>0 .{ }^{13}$ Using (8), we first notice that the left hand side of equation (21) increases with respect to $A$. Furthermore, $\lim _{A \rightarrow 0}[1-\alpha(\operatorname{Dm}(\bar{a}, \bar{K}) A R(\bar{a}))] A=0$. Therefore, under the assumption:

Assumption $3 \lim _{A \rightarrow+\infty}[1-\alpha(\operatorname{Dm}(\bar{a}, \bar{K}) A R(\bar{a}))] A>1 /(m(\bar{a}, \bar{K}) W(\bar{a}))$.

there exists a unique solution $A^{*}$ to the equation:

$$
\left[1-\alpha\left(D m(\bar{a}, \bar{K}) A^{*} R(\bar{a})\right)\right] A^{*}=\frac{1}{m(\bar{a}, \bar{K}) W(\bar{a})}
$$

Especially, this last equation is satisfied for $D=D^{*}$, with:

$$
D^{*}=1 /\left[m(\bar{a}, \bar{K}) A^{*} R(\bar{a})\right]
$$

Given $A^{*}$ and $D^{*}$, one can easily see that there exists a unique solution $B^{*}>0$ to the equation:

$$
B^{*}=\left(1 / D^{*}\right) U^{*}(1) m(\bar{a}, \bar{K}) A^{*} W(\bar{a}) / v^{\prime}(\bar{K} / \bar{a})
$$

Proposition 1 Under Assumptions 1-3, $(K, a)=(\bar{K}, \bar{a})$ is a stationary solution of the dynamic system (19)-(20) if and only if $A, B$ and $D$ are the unique solutions of (23), (24) and (25).

Note that for $D=D^{*}$, we have $\alpha(1), \eta(1)$ and $U^{*}(1)$ at the steady state, i.e., these three functions do not depend on the steady state.

Before studying local dynamics, it is useful to define the following relationships. First, we note $s(a) \equiv f^{\prime}(a) a / f(a) \in(0,1)$ the capital share in production. $^{14}$ Moreover, if we note $\sigma(a)$ the elasticity of substitution between capital and labor, $1 / \sigma(a)=d \ln W(a) / d \ln a-d \ln R(a) / d \ln a$. Since $W^{\prime}(a)=-a R^{\prime}(a)$, we obtain:

$$
W^{\prime}(a) a / W(a)=s(a) / \sigma(a) \text { and } R^{\prime}(a) a / R(a)=-(1-s(a)) / \sigma(a)
$$

\footnotetext{
${ }^{12}$ Since we will study local dynamics around the steady state $(K, a)=(\bar{K}, \bar{a}), N>2$ is required at this steady state, which is equivalent to $f(\bar{a}) \bar{K} /(\phi \bar{a})>\varepsilon(2-\tau)+\tau$.

${ }^{13}$ Note that the positive and finite levels of $\bar{K}$ and $\bar{a}$ can be chosen arbitrarily. The main advantage of using this method to establish the existence of a steady state is the following. In the next section where we study local dynamics, a modification of an elasticity, evaluated at the steady state, will not change the steady state levels $\bar{K}$ and $\bar{a}$ ensured by $A, B$ and $D$.

${ }^{14}$ Using (13) and (16), we can show that $\rho_{t} k_{t-1} / y_{t}=f^{\prime}\left(a_{t}\right) a_{t} / f\left(a_{t}\right) \equiv s\left(a_{t}\right)$.
} 
We also compute the elasticities of the inverse of the mark-up factor $m(a, K)$ with respect to $K$ and $a$ :

$$
\begin{aligned}
\varepsilon_{m, K}(a, K) & \equiv \frac{\partial m(a, K)}{\partial K} \frac{K}{m(a, K)}=\frac{\tau \phi a}{f(a) K-\tau \phi a} \\
\varepsilon_{m, a}(a, K) & \equiv \frac{\partial m(a, K)}{\partial a} \frac{a}{m(a, K)}=-(1-s(a)) \varepsilon_{m, K}(a, K)
\end{aligned}
$$

Note that without strategic interaction $(\tau=0)$, we obviously have that $\varepsilon_{m, K}(a, K)=\varepsilon_{m, a}(a, K)=0$, because the mark-up is constant. With strategic interactions between the producers $(\tau=1)$, both the capital and labor affect positively the inverse of the mark-up. Indeed, since $a=K / L$, these two effects are measured by $\varepsilon_{m, K}(a, K)+\varepsilon_{m, a}(a, K)=s(a) \varepsilon_{m, K}(a, K)>0$ and $-\varepsilon_{m, a}(a, K)=(1-s(a)) \varepsilon_{m, K}(a, K)>0$ respectively. Since both these expressions linearly depend on $\varepsilon_{m, K}(a, K)$, this last term will be interpreted as a measure of the degree of mark-up variability in the rest of the paper.

\section{Local Dynamics}

We study local dynamics to analyze the occurrence of local indeterminacy and endogenous cycles. To underline the role of strategic interactions and mark-up variability on the dynamics, we consider first the model without strategic interaction characterized by a constant mark-up $(\tau=0)$. In a second step, we analyze the dynamics when there are strategic interactions and a variable mark-up $(\tau=1)$. We will see that the substitution between capital and labor will play a key role on the stability properties of the steady state.

Log-linearizing the dynamic system (19)-(20) in the neighborhood of the steady state $(K, a)=(\bar{K}, \bar{a})$, we get:

Proposition 2 Note $\alpha \equiv \alpha(1), \eta \equiv \eta(1), s \equiv s(\bar{a}), \sigma \equiv \sigma(\bar{a}), \varepsilon_{m, K} \equiv$ $\varepsilon_{m, K}(\bar{a}, \bar{K})$ and $\varepsilon_{v} \equiv \varepsilon_{v}(\bar{K} / \bar{a}) .{ }^{15}$ Under Assumptions 1-3, the characteristic polynomial is defined by $P(\lambda) \equiv \lambda^{2}-\operatorname{Tr} \lambda+$ Det $=0$, where the trace $\operatorname{Tr}$ and the determinant Det of the associated Jacobian matrix are respectively given

\footnotetext{
${ }^{15}$ Notice that many elasticities that enter the characteristic polynomial depend on the steady state $(\bar{K}, \bar{a})$. Since we study dynamics in the neighborhood of this steady state and the steady state levels of capital $\bar{K}$ and capital-labor ratio $\bar{a}$ are ensured by the scaling parameters $A, B$ and $D$, these elasticities can be considered as parameters.
} 
by:

$$
\begin{aligned}
\operatorname{Tr} & =\frac{\sigma \varepsilon_{m, K}(\alpha s-1)+(1-\alpha \eta) \varepsilon_{m, K}+s+(1-\alpha)(1-s)}{(1-\alpha)(1-s)\left(\sigma \varepsilon_{m, K}+1\right)} \\
& +\varepsilon_{v} \frac{\sigma\left(1+s \alpha(1-\eta) \varepsilon_{m, K}\right)-\alpha(1-\eta)(1-s)}{(1-\alpha)(1-s)\left(\sigma \varepsilon_{m, K}+1\right)} \\
\text { Det } & =\frac{s}{(1-s)(1-\alpha)}\left(1+\varepsilon_{v}\right)
\end{aligned}
$$

Proof. See the Appendix.

The characteristic polynomial $P(\lambda)$ is useful to analyze the local stability (indeterminacy) of the steady state and the occurrence of bifurcations. Evaluating $P(\lambda)$ at $\lambda=-1,0$ and 1 , the steady state is locally indeterminate when $P(1)>0, P(-1)>0$ and $P(0)<1$. In this case, the two eigenvalues have a modulus smaller than one and fluctuations coming from the volatility of expectations can occur in a neighborhood of the steady state. In all other cases, the steady state is determinate, being a saddle if $P(1)>0>P(-1)$ or $P(-1)>0>P(1)$ and a source otherwise.

Moreover, following the continuous change of a parameter, when $P(1)=$ 0 , one eigenvalue crosses the value 1 and there is generically an exchange of stability between two steady states, through the occurrence of a transcritical bifurcation. When $P(-1)=0$, one eigenvalue crosses the value -1 and a cycle of period 2 generically appears, through the occurrence of a flip bifurcation. When $P(1)>0, P(-1)>0$ and $P(0)=1$, the two eigenvalues are complex conjugates and have a modulus crossing the value 1 . Therefore, an invariant closed curve generically appears around the steady state, through a Hopf bifurcation. ${ }^{16}$

We will now use this methodology to analyze the occurrence of endogenous fluctuations in our model. To be able to make clear the role of strategic interactions on the dynamics, we begin by briefly considering the case where producers behave as in the so-called Dixit and Stiglitz (1977) model, i.e., the framework without strategic interaction and mark-up variability.

\subsection{No Strategic Interaction and Constant Mark-up}

Without strategic interaction, we have $\tau=0$. Therefore, $\varepsilon_{m, K}=0$, i.e., the mark-up factor is constant and equal to $\varepsilon /(\varepsilon-1)$. The stability properties of the steady state are summarized in the following proposition:

\footnotetext{
${ }^{16}$ As it is shown by Grandmont, Pintus, and de Vilder (1998), when there is one predetermined and one non-predetermined variable, cycles are subject to sunspot equilibria if they are locally stable.
} 
Proposition 3 Under Assumptions 1-3, a constant mark-up $(\tau=0), s<$ $1 / 2$ and $\alpha<(1-2 s) /(1-s)$, the following generically holds.

1. When $\eta \geq 1-s /[\alpha(1-s)]$ and $\sigma<\alpha(1-\eta)(1-s)+s$, the steady state is locally indeterminate for $\varepsilon_{v}<\varepsilon_{v_{H}}$.

2. When $\eta<1-s /[\alpha(1-s)]$,

(i) if $\sigma<\alpha(1-\eta)(1-s)-s$, the steady state is locally indeterminate for $\varepsilon_{v}<\min \left\{\varepsilon_{v_{H}}, \varepsilon_{v_{F}}\right\}$

(ii) if $\sigma \in(\alpha(1-\eta)(1-s)-s, \alpha(1-\eta)(1-s)+s)$, the steady state is locally indeterminate for $\varepsilon_{v}<\varepsilon_{v_{H}}$.

In all other cases, the steady state is a saddle or a source, i.e., locally determinate. Moreover, when $\varepsilon_{v}$ crosses the value $\varepsilon_{v_{H}}$, a Hopf bifurcation generically occurs, while when $\varepsilon_{v}$ crosses the value $\varepsilon_{v_{F}}$, a flip bifurcation generically occurs, with:

$$
\varepsilon_{v_{H}} \equiv(1-\alpha)(1-s) / s-1 \text { and } \varepsilon_{v_{F}} \equiv \frac{2[s+(1-\alpha)(1-s)]}{\alpha(1-\eta)(1-s)-s-\sigma}
$$

Proof. See the Appendix.

In the absence of strategic interaction, i.e., under a constant mark-up, local indeterminacy and endogenous cycles require a weak elasticity of capitallabor substitution $(\sigma<\alpha(1-\eta)(1-s)+s)$ and a sufficiently elastic labor supply $\left(1 / \varepsilon_{v}\right)$. Note that these results are closely related to those obtained under perfect competition. ${ }^{17}$ Indeed, under monopolistic competition, endogenous product diversity and a constant mark-up, imperfect competition has no influence on local dynamics. We have $\varepsilon_{m, K}=0$ and, by direct inspection of equations (29) and (30), neither the mark-up factor $\epsilon /(\epsilon-1)$ nor the fixed cost $\phi$ affect the determinant Det and the trace $\operatorname{Tr}$ of the Jacobian matrix.

Especially, when the propensity to consume at the first period of life is zero $(\alpha=0)$, we recover the results obtained by Reichlin (1986). The occurrence of endogenous fluctuations requires an elasticity of capital-labor substitution smaller than the capital share in income, which needs to be smaller than one half $(\sigma<s<1 / 2) .{ }^{18}$ However, when the propensity to save becomes smaller than $1(\alpha>0)$, the elasticity of intertemporal substitution in consumption has a role on the stability properties. When there is

\footnotetext{
${ }^{17}$ See Reichlin (1986), Cazzavillan and Pintus (2004) and Nourry and Venditti (2006).

${ }^{18}$ Note that we obtain the same conclusion when $\alpha>0$ but $\eta=1$.
} 
intertemporal substitutability $(\eta>1)$, indeterminacy emerges for a smaller range of elasticities of capital-labor substitution, whereas intertemporal complementarity $(\eta<1)$ increases the range of the elasticity $\sigma$ for indeterminacy.

To give now an intuitive interpretation of these results, recall that the dynamics are defined by the two following equations:

$$
\begin{aligned}
K_{t} & =\left(1-\alpha\left(D \rho_{t+1}\right)\right) \omega_{t} L_{t} \\
(1 /(B D)) U^{*}\left(D \rho_{t+1}\right) \omega_{t} & =v^{\prime}\left(L_{t}\right)
\end{aligned}
$$

To explain the occurrence of fluctuations driven by self-fulfilling expectations, consider first that savings do not depend on the future real interest rate $(\eta=1)$ or that there is a unit propensity to save $(\alpha=0)$. Assume that consumers expect a higher future real interest rate $\rho_{t+1}$. From (33), they increase their labor supply $\left(\left(d L_{t} / d \rho_{t+1}\right)\left(\rho_{t+1} / L_{t}\right)=(1-\alpha) / \varepsilon_{v}>0\right)$. Since the mark-up is constant, the labor income $\omega_{t} L_{t}$ decreases only if the capitallabor substitution is sufficiently small $(1-s / \sigma<0)$. In this case, savings $K_{t}$ decrease (see (32)), implying an increase of the future real interest rate $\rho_{t+1}$. We deduce that two effects are crucial for expectation driven fluctuations: the effect of the future real interest rate on the current labor supply and the negative effect of labor supply on labor income. The first one is sufficiently strong if $\varepsilon_{v}$ is small enough and the second one requires $\sigma<s$.

Assuming now that households consume in both period $(0<\alpha<1)$ and have a non-unit elasticity of intertemporal substitution in consumption $(\eta \neq 1)$, there is an adding effect. Indeed, if the elasticity of intertemporal substitution in consumption is greater than one $(\eta>1)$, the final increase of the future real interest rate raises savings through its positive effect on the propensity to save $\left(1-\alpha\left(D \rho_{t+1}\right)\right)$. Therefore, the decrease of capital $K_{t}$ and, hence, the emergence of expectation driven fluctuations requires a lower substitution between capital and labor. On the contrary, if the elasticity of intertemporal substitution in consumption is smaller than one $(\eta<1)$, endogenous fluctuations can occur for an elasticity of capital-labor substitution greater than $s$, because the propensity to save is decreasing in the future real interest rate. Finally, note that this economic intuition does not only allows us to recover the main conclusions of Proposition 3, but also underlines that, without strategic interaction, imperfect competition has no role on the occurrence of expectation driven fluctuations.

We consider now the case with strategic interactions between producers and mark-up variability $\left(\varepsilon_{m, K} \neq 0\right)$. We will see that, in this case, endogenous fluctuations can occur when capital and labor are not weak substitutes. 


\subsection{Strategic Interactions and Variable Mark-up}

When there are strategic interactions, we have $\tau=1$ and the mark-up factor is counter-cyclical $\left(\varepsilon_{m, K}>0\right)$. In the next proposition, we analyze the implications of such a market power variability on local indeterminacy and endogenous cycles. For simplification, we focus on the case where labor supply is infinitely elastic $\left(\varepsilon_{v}=0\right) .{ }^{19}$

Proposition 4 Under Assumptions 1-3, a variable mark-up $(\tau=1)$, an infinitely elastic labor supply $\left(\varepsilon_{v}=0\right), s<1 / 2$ and $\alpha<(1-2 s) /(1-s)$, the following generically holds.

1. When $\eta<1 / \alpha$, the steady state is locally indeterminate if $\sigma_{T}<\sigma<\sigma_{F}$.

2. When $\eta \geq 1 / \alpha$, the steady state is locally indeterminate if $\varepsilon_{m, K}<$ $2[s+(1-\alpha)(1-s)] /(\alpha \eta-1)$ and $\sigma<\sigma_{F}$.

In all other cases, the steady state is locally determinate. Moreover, when $\sigma$ crosses the value $\sigma_{T}$, a transcritical bifurcation generically occurs, while when $\sigma$ crosses the value $\sigma_{F}$, a flip bifurcation generically occurs, with:

$$
\sigma_{T} \equiv \frac{1-\alpha \eta}{2-\alpha} \text { and } \sigma_{F} \equiv \frac{(1-\alpha \eta) \varepsilon_{m, K}+2[s+(1-\alpha)(1-s)]}{\alpha \varepsilon_{m, K}(1-2 s)}
$$

Proof. See the Appendix.

When there are strategic interactions between producers and a variable mark-up, fluctuations due to self-fulfilling expectations and endogenous cycles emerge for high values of the elasticity of capital-labor substitution. ${ }^{20}$ Hence, this result is in contrast to the one obtained in the previous subsection where the monopolistic competition model without strategic interaction is studied and endogenous fluctuations require a sufficiently small elasticity $\sigma$ (see Proposition 3). Therefore, strategic interactions have a key role on the dynamics, promoting local indeterminacy when capital and labor are not weak substitutes.

We also note that the configuration considered in several previous papers,${ }^{21}$ where households do not consume at their first period of life, i.e., $\alpha=0$, seems to be quite specific. The upper bound $\sigma_{F}$ disappears and case

\footnotetext{
${ }^{19}$ Since Hansen (1985), this assumption is often introduced in macroeconomic dynamic models.

${ }^{20}$ Notice that these results are obtained assuming an infinitely elastic labor supply. By continuity, they are still relevant for a sufficiently elastic labor supply.

${ }^{21}$ One can refer to Reichlin (1986), Cazzavillan (2001) and Dos Santos Ferreira and Lloyd-Braga (2005).
} 
1 of Proposition 4 applies: local indeterminacy occurs for all $\sigma>1 / 2{ }^{22}$ Moreover, the range of capital-labor substitution for indeterminacy does not depend on the degree of mark-up variability, measured by $\varepsilon_{m, K}$. However, the results are different when the propensity to consume at the first period is strictly positive $(\alpha>0)$.

Corollary 1 Under Assumptions 1-3, a variable mark-up $(\tau=1)$, an infinitely elastic labor supply $\left(\varepsilon_{v}=0\right), s<1 / 2$ and $(1-2 s) /(1-s)>\alpha>0$, the range for indeterminacy of the elasticity of capital-labor substitution $\sigma$ is decreasing in $\varepsilon_{m, K}$. Moreover, when $\sigma>(1-\alpha \eta) /[\alpha(1-2 s)]$ and $\varepsilon_{m, K}$ increases, the steady state is locally indeterminate for $\varepsilon_{m, K}<\varepsilon_{m, K}^{F}$, a flip bifurcation generically occurs for $\varepsilon_{m, K}=\varepsilon_{m, K}^{F}$, and the steady state is a saddle for $\varepsilon_{m, K}>\varepsilon_{m, K}^{F}$, with:

$$
\varepsilon_{m, K}^{F} \equiv 2 \frac{s+(1-\alpha)(1-s)}{\alpha \eta-1+\alpha(1-2 s) \sigma}
$$

Proof. See the Appendix.

This corollary shows that a larger degree of mark-up variability, through a higher level of $\varepsilon_{m, K}$, reduces the range for indeterminacy of the elasticity of capital-labor substitution. Furthermore, when the level of mark-up variability is high enough, indeterminacy can even be ruled out: a locally indeterminate steady state can become a saddle, i.e., determinate. It is however worth to note that cycles of period two generically occur, through a flip bifurcation, for a weaker substitution between capital and labor when the degree of mark-up variability is higher. ${ }^{23}$

Because $\varepsilon_{m, K}=\phi \bar{a} /[f(\bar{a}) \bar{K}-\phi \bar{a}]$ (see equation (27)), there is a positive relationship between the degree of mark-up variability and the level of the fixed cost $\phi$, i.e., the level of increasing returns. From Corollary 1, we deduce that smaller (increasing) returns to scale raise the range for indeterminacy of the elasticity of capital-labor substitution. This also means that the results obtained in this paper do not require large increasing returns. ${ }^{24}$ This is in accordance with several empirical studies (Basu and Fernald (1995), Burnside (1996), Basu and Fernald (1997)) underlining that too large increasing returns are not admissible.

We give now an economic interpretation of our results. For simplification, we consider the case of a unit elasticity of intertemporal substitution in

\footnotetext{
${ }^{22}$ Using (34), we notice that when $\alpha$ tends to $0, \sigma_{T}$ tends to $1 / 2$ and $\sigma_{F}$ to $+\infty$.

${ }^{23}$ Note that $\sigma_{F}$ is decreasing from $+\infty$ when $\varepsilon_{m, K}$ is increasing from 0 (see (34)).

${ }^{24}$ Cazzavillan (2001) and Lloyd-Braga, Nourry, and Venditti (2007) obtain related conclusions in overlapping generations models where increasing returns are due to productive externalities.
} 
consumption $(\eta=1)$, implying that the propensity to save does not depend on the future real interest rate. Then, the dynamics are governed by the two following equations:

$$
\begin{aligned}
K_{t} & =(1-\alpha) \omega_{t} L_{t} \\
(1 /(B D)) U^{*}\left(D \rho_{t+1}\right) \omega_{t} & =v^{\prime}\left(L_{t}\right)
\end{aligned}
$$

It is useful to recall that the real wage and the real interest rate are affected by the inverse of mark-up factor, increasing in aggregate capital and labor because of strategic interactions between producers. Moreover, under the assumptions of Proposition 4, we have $\sigma_{T}>s$, i.e., the labor income $\omega_{t} L_{t}$ is increasing in $L_{t}$ for all $\sigma>\sigma_{T}$, even if the degree of mark-up variability is arbitrarily small. The economic intuition we give now is based on the existence of non monotonic trajectories of the capital stock. Assume that we deviate from the steady state by an increase of capital $K_{t-1}$. This raises labor income $\omega_{t} L_{t}$ and, therefore, future capital $K_{t}$ (see (36)). Following such a sequence, consumers expect a decrease of the future real interest rate $\rho_{t+1}$. From (37), they decrease their current labor supply. This feedback effect negatively affects the labor income. Hence, a non monotonic trajectory of capital emerges if this second effect coming from consumers' expectations dominates. This is possible if the mark-up is variable because, in this case, a variation of aggregate labor has two effects on the real wage: the usual one through the marginal productivity of labor and a second one through the inverse of mark-up. Indeed, following a decrease of labor, aggregate production reduces, which has a negative effect on the product diversity. Since each producer has less competitors, it increases is mark-up, which negatively affects the real wage.

To conclude this economic intuition, note that the capital path initially due to a deviation from the steady state has to be locally stable. Therefore, even if the feedback effect, through which both the labor supply and the labor income reduce, has to dominate, it should not be too large because, otherwise, some form of instability emerges and the steady state is determinate. Since the elasticity of labor income with respect to labor can be given by $(\partial \omega L / \partial L)(L / \omega L)=1+(1-s) \varepsilon_{m, K}-s / \sigma$, this occurs especially if the degree of mark-up variability is sufficiently large and inputs are high substitutes (see Corollary 1).

We conclude this section by providing a numerical illustration of our findings and underline that endogenous fluctuations occur under more relevant degrees of capital-labor substitution when producers behave strategically and mark-up is variable. To do that, we choose a usual value for the capital share in income, $s=1 / 3$. To satisfy the assumption $\alpha<(1-2 s) /(1-s)$, we set 
$\alpha=0.4$ and, as in Proposition 4 and Corollary 1, we restrict our attention to $\varepsilon_{v}=0$, which could be justified by indivisible labor. Furthermore, several empirical studies find small values for the elasticity of intertemporal substitution in consumption. However, in our overlapping generations model, this implies that savings are decreasing in the future real interest rate, whereas the opposite relationship between savings and the real interest rate seems to be more reasonable. To avoid this difficulty, we assume $\eta=1$.

Using this parametrization, we can easily see that, without strategic interaction and mark-up variability, the occurrence of indeterminacy requires $\sigma<1 / 3$ (see Proposition 3), i.e., a too weak elasticity of capital-labor substitution to be in accordance with empirical studies (see Duffy and Papageorgiou (2000)). In the presence of strategic interactions and mark-up variability, case 1 of Proposition 4 applies, i.e., local indeterminacy requires $\sigma_{T}<\sigma<\sigma_{F}$. Evaluating the lower bound, we get $\sigma_{T}=0.375$. Taking as given the degree of mark-up variability $\varepsilon_{m, K}$, the upper bound is given by $\sigma_{F}=4.5+11 / \varepsilon_{m, K}$. To determine now the admissible values for $\varepsilon_{m, K}$, recall that the number of producers has to be strictly greater than 2 around the steady state. This implies that $f(\bar{a}) \bar{K} /(\phi \bar{a})>1+\varepsilon$. Using this inequality and equation (27),

we obtain $\varepsilon_{m, K}<1 / \varepsilon<1$. Therefore, when $\varepsilon_{m, K}$ increases from 0 , the upper bound $\sigma_{F}$ decreases from $+\infty$, but is always strictly greater than 15.5 . We conclude that with strategic interactions and mark-up variability, indeterminacy occurs for a large range of elasticities of capital-labor substitution, that encompasses the Cobb-Douglas case $(\sigma=1)$ and all the empirically relevant values (see Duffy and Papageorgiou (2000)).

\section{Conclusion}

In macroeconomics, the Dixit and Stiglitz (1977) monopolistic competition model is the most commonly used framework to introduce imperfect competition on product markets. As it is well known, this model rules out strategic interactions between producers. In this paper, we stress that this approach of imperfect competition can be misleading to understand the role of product market imperfections on expectation driven fluctuations.

We analyze a model that encompasses the Dixit and Stiglitz (1977) framework as a particular case, but also allows us to consider the case where producers have strategic interactions (Yang and Hejdra (1993)). Since the product diversity is endogenously determined by a zero profit condition, the mark-up factor is variable under strategic behaviors, whereas it is constant in the Dixit and Stiglitz (1977) approach. Using an overlapping generations model, we show that this mark-up variability significantly affects the dy- 
namic properties of the steady state. Indeed, in contrast to the configuration without strategic interaction, indeterminacy does not require a too weak elasticity of capital-labor substitution when the mark-up is variable, but occurs for a large and plausible range of this elasticity. Therefore, strategic behaviors of producers seem to play a key role to understand the occurrence of endogenous fluctuations.

More generally, our conclusions suggest that taking into account imperfectly competitive behaviors of firm still constitutes an important issue to understand macroeconomic fluctuations, as soon as one considers a significant departure from the perfect competition.

\section{Appendix}

The existence of $\alpha\left(D \rho_{t+1}\right)$

The first order condition of the utility maximization can be written:

$$
\frac{(1 / D) U_{1}\left(C_{1 t} / D, C_{2 t+1}\right)}{U_{2}\left(C_{1 t} / D, C_{2 t+1}\right)}=\rho_{t+1}
$$

Since $U$ is homogeneous of degree $1, U_{1}$ and $U_{2}$ are homogeneous of degree 0 . Therefore, equation (38) is equivalent to:

$$
g\left(C_{1 t} /\left(D C_{2 t+1}\right)\right) \equiv \frac{U_{1}\left(C_{1 t} /\left(D C_{2 t+1}\right), 1\right)}{U_{2}\left(1,\left(D C_{2 t+1}\right) / C_{1 t}\right)}=D \rho_{t+1}
$$

where $g$ is a decreasing function. We deduce that there exists an inverse function $g^{-1}$ such that $C_{1 t}=D g^{-1}\left(D \rho_{t+1}\right) C_{2 t+1}$. Substituting this equation into the lifetime budget constraint $C_{1 t}+C_{2 t+1} / \rho_{t+1}=\omega_{t} L_{t}$, we obtain:

$$
\alpha\left(D \rho_{t+1}\right)=\frac{D \rho_{t+1} g^{-1}\left(D \rho_{t+1}\right)}{1+D \rho_{t+1} g^{-1}\left(D \rho_{t+1}\right)}
$$

Elasticity of Intertemporal Substitution in Consumption

We define:

$$
\frac{C_{1 t}}{D C_{2 t+1}}=\frac{\alpha\left(D \rho_{t+1}\right)}{\left(1-\alpha\left(D \rho_{t+1}\right)\right) D \rho_{t+1}} \equiv H\left(D \rho_{t+1}\right)
$$


The elasticity of intertemporal substitution in consumption $\eta\left(D \rho_{t+1}\right)$ is determined by the elasticity of $H$, in absolute value:

$$
\eta\left(D \rho_{t+1}\right)=-\frac{H^{\prime}\left(D \rho_{t+1}\right) D \rho_{t+1}}{H\left(D \rho_{t+1}\right)}
$$

After some computations, we obtain the expression given in (8).

Definition and Elasticity of $U^{*}$

Substituting (5) and (6) into the function $U\left(C_{1 t} / D, C_{2 t+1}\right)$ times $D$, we define $U^{*}\left(D \rho_{t+1}\right)$ as follows:

$$
U\left(\alpha\left(D \rho_{t+1}\right),\left(1-\alpha\left(D \rho_{t+1}\right)\right) D \rho_{t+1}\right) \omega_{t} L_{t} \equiv U^{*}\left(D \rho_{t+1}\right) \omega_{t} L_{t}
$$

Note that both sides of this equation linearly depend on the wage income. Furthermore, using the first order condition $U_{1}=U_{2} D \rho_{t+1}{ }^{25}$ and the Euler identity $U\left(\alpha,(1-\alpha) D \rho_{t+1}\right)=U_{1} \alpha+U_{2}(1-\alpha) D \rho_{t+1}$, we have:

$$
U^{*^{\prime}} D \rho_{t+1}=\left(U_{1}-U_{2} D \rho_{t+1}\right) \alpha^{\prime} D \rho_{t+1}+U_{2} D \rho_{t+1}(1-\alpha)=U^{*}(1-\alpha)>0
$$

We deduce that the elasticity of $U^{*}\left(D \rho_{t+1}\right)$ is given by $1-\alpha\left(D \rho_{t+1}\right)$.

\section{Proof of Proposition 2}

To get the characteristic polynomial, we log-linearize the dynamic system (19)-(20) in the neighborhood of the steady state $(K, a)=(\bar{K}, \bar{a})$. Using equations $(8),(26),(27),(28)$ and the elasticity of $U^{*}\left(D \rho_{t+1}\right)$ evaluated at the steady state, we obtain:

$$
\begin{aligned}
\frac{d K_{t}}{K} & =J_{11} \frac{d K_{t-1}}{K}+J_{12} \frac{d a_{t}}{a} \\
\frac{d a_{t+1}}{a} & =J_{21} \frac{d K_{t-1}}{K}+J_{22} \frac{d a_{t}}{a}
\end{aligned}
$$

\footnotetext{
${ }^{25}$ In order to simplify the notations, we do not write the arguments of the functions when this is not confusing and we note $U_{i} \equiv \partial U\left(x_{1}, x_{2}\right) / \partial x_{i}, i=1,2$.
} 
with:

$$
\begin{aligned}
J_{11} & =1+\varepsilon_{m, K}+\frac{\alpha(1-\eta)}{1-\alpha}\left(\varepsilon_{m, K}-\varepsilon_{v}\right) \\
J_{12} & =-(1-s) \varepsilon_{m, K}-1+\frac{s}{\sigma}+\frac{\alpha(1-\eta)}{1-\alpha}\left[\varepsilon_{v}+\frac{s}{\sigma}-(1-s) \varepsilon_{m, K}\right] \\
J_{21} & =\frac{\varepsilon_{m, K}\left(1+\varepsilon_{m, K}\right)}{(1-s)\left(\varepsilon_{m, K}+1 / \sigma\right)}+\frac{\left[1+\alpha(1-\eta) \varepsilon_{m, K}\right]\left(\varepsilon_{m, K}-\varepsilon_{v}\right)}{(1-\alpha)(1-s)\left(\varepsilon_{m, K}+1 / \sigma\right)} \\
J_{22} & =-\frac{\varepsilon_{m, K}}{(1-s)\left(\varepsilon_{m, K}+1 / \sigma\right)}\left[(1-s) \varepsilon_{m, K}+1-\frac{s}{\sigma}\right] \\
+ & \frac{1+\alpha(1-\eta) \varepsilon_{m, K}}{(1-\alpha)(1-s)\left(\varepsilon_{m, K}+1 / \sigma\right)}\left[\varepsilon_{v}+\frac{s}{\sigma}-(1-s) \varepsilon_{m, K}\right]
\end{aligned}
$$

As it is well known, the trace $\operatorname{Tr}$ and the determinant Det of the associated Jacobian matrix are defined by $T r=J_{11}+J_{22}$ and Det $=J_{11} J_{22}-J_{12} J_{21}$. Using equations (45) - (48), we obtain (29) and (30).

\section{Proof of Proposition 3}

When $\varepsilon_{v}$ tends to $0, P(0)=\operatorname{Det}$ is strictly smaller than 1 because $s<1 / 2$ and $\alpha<(1-2 s) /(1-s)$. We deduce that because Det is increasing in $\varepsilon_{v}$, Det is strictly smaller than 1 if $\varepsilon_{v}<\varepsilon_{v_{H}}$, equal to 1 if $\varepsilon_{v}=\varepsilon_{v_{H}}$, and strictly greater than 1 if $\varepsilon_{v}>\varepsilon_{v_{H}}$, where the critical value $\varepsilon_{v_{H}}$ is given in the proposition.

Since $\tau=0$, we have $\varepsilon_{m, K}=0$. Using equations (29) and (30), $P(1)=$ $1-T+D$ and $P(-1)=1+T+D$ are respectively equal to:

$$
\begin{aligned}
P(1) & =\varepsilon_{v} \frac{s+\alpha(1-\eta)(1-s)-\sigma}{(1-\alpha)(1-s)} \\
P(-1) & =\frac{2(s+(1-\alpha)(1-s))+\varepsilon_{v}(\sigma+s-\alpha(1-\eta)(1-s))}{(1-\alpha)(1-s)}
\end{aligned}
$$

We can notice that $P(1)>0$ if and only if $\sigma<\alpha(1-\eta)(1-s)+s$, and $P(1) \leq 0$ otherwise. When $\eta \geq 1-s /[\alpha(1-s)], P(-1)$ is strictly positive for all $\sigma>0$ and $\varepsilon_{v}>0$. We deduce that when $\eta \geq 1-s /(\alpha(1-s))$, the occurrence of local indeterminacy requires $\sigma<\alpha(1-\eta)(1-s)+s$ and $\varepsilon_{v}<\varepsilon_{v_{H}}$.

Considering now that $\eta<1-s /[\alpha(1-s)]$, there are three subcases:

- If $\sigma<\alpha(1-\eta)(1-s)-s, P(1)>0$ and $P(-1)>0$ if $\varepsilon_{v}<\varepsilon_{v_{F}}$, where the critical value $\varepsilon_{v_{F}}$ is given in the proposition. Therefore, the steady state is locally indeterminate for $\varepsilon_{v}<\min \left\{\varepsilon_{v_{H}}, \varepsilon_{v_{F}}\right\}$; 
- If $\sigma \in(\alpha(1-\eta)(1-s)-s, \alpha(1-\eta)(1-s)+s), P(1)>0$ and $P(-1)>0$ for all $\varepsilon_{v}>0$. Therefore, the steady is locally indeterminate for $\varepsilon_{v}<\varepsilon_{v_{H}}$;

- If $\sigma>\alpha(1-\eta)(1-s)+s, P(1)<0$ and $P(-1)>0$ for all $\varepsilon_{v}>0$. Then, the steady state is always a saddle, i.e., locally determinate.

\section{Proof of Proposition 4}

Under $\varepsilon_{v}=0$, Det belongs to $(0,1)$ because $s<1 / 2$ and $\alpha<(1-2 s) /(1-$ $s)$ (see equation (30)). Furthermore, using equations (29) and (30), we are able to determine $P(1)$ and $P(-1)$ :

$$
\begin{aligned}
P(1) & =\varepsilon_{m, K} \frac{\sigma(2-\alpha)-(1-\alpha \eta)}{(1-\alpha)(1-s)\left(\sigma \varepsilon_{m, K}+1\right)} \\
P(-1) & =\frac{(1-\alpha \eta) \varepsilon_{m, K}+2[s+(1-\alpha)(1-s)]-\sigma \alpha \varepsilon_{m, K}(1-2 s)}{(1-\alpha)(1-s)\left(\sigma \varepsilon_{m, K}+1\right)}
\end{aligned}
$$

We deduce that when $\eta<1 / \alpha, P(1)>0$ if and only if $\sigma>\sigma_{T}$ and $P(-1)>0$ if and only if $\sigma<\sigma_{F}$, where the critical values $\sigma_{T}$ and $\sigma_{F}$ are given in the proposition. Therefore, local indeterminacy occurs for $\sigma_{T}<\sigma<\sigma_{F}$.

When $\eta \geq 1 / \alpha, P(1)>0$ for all $\sigma>0$. Moreover, when $\varepsilon_{m, K} \geq 2[s+$ $(1-\alpha)(1-s)] /(\alpha \eta-1), P(-1)<0$ for all $\sigma$. On the contrary, when $\varepsilon_{m, K}<$ $2[s+(1-\alpha)(1-s)] /(\alpha \eta-1), P(-1)>0$ if $\sigma<\sigma_{F}$ and $P(-1) \leq 0$ otherwise. Hence, local indeterminacy requires $\varepsilon_{m, K}<2[s+(1-\alpha)(1-s)] /(\alpha \eta-1)$ and $\sigma<\sigma_{F}$ in this case.

\section{Proof of Corollary 1}

We begin by noting that Det does not depend on $\varepsilon_{m, K}$ and belongs to $(0,1)$ because, as in Proposition $4, \varepsilon_{v}=0, s<1 / 2$ and $\alpha<(1-2 s) /(1-s)$. Then, the first part of the corollary is proved noting that $\sigma_{T}$ does not depend on $\varepsilon_{m, K}$ and $\sigma_{F}$ decreases with respect to $\varepsilon_{m, K}$.

In order to show the second part of the corollary, we notice that for $\sigma>(1-\alpha \eta) /[\alpha(1-2 s)]$, we have $P(1)>0$. Moreover, $P(-1)>0$ if $\varepsilon_{m, K}<\varepsilon_{m, K}^{F}, P(-1)=0$ if $\varepsilon_{m, K}=\varepsilon_{m, K}^{F}$, and $P(-1)<0$ if $\varepsilon_{m, K}>\varepsilon_{m, K}^{F}$, where the critical value $\varepsilon_{m, K}^{F}$ is given in the corollary. 


\section{References}

Aloi, M., H. D. Dixon, And T. Lloyd-Braga (2000): "Endogenous Fluctuations in an Open Economy with Increasing Returns to Scale," Journal of Economic Dynamics and Control, 24, 97-125.

BAsu, S., And J. Fernald (1995): "Are Apparent Productive Spillovers a Figment of Specification Error ?," Journal of Monetary Economics, 36, $165-188$.

(1997): "Returns to Scale in US Production: Estimates and Implications," Journal of Political Economy, 105, 249-283.

Benassy, J.-P. (1996): "Taste for Variety and Optimum Production Patterns in Monopolistic Competition," Economics Letters, 52, 41-47.

BiLs, M. (1987): "The Cyclical Behavior of Marginal Cost and Price," American Economic Review, 77, 838-855.

Burnside, C. (1996): "Production Function Regressions, Returns to Scale, and Externalities," Journal of Monetary Economics, 37, 177-201.

Cazzavillan, G. (2001): "Indeterminacy and Endogenous Fluctuations with Arbitrarily Small Externalities," Journal of Economic Theory, 101, $133-157$.

Cazzavillan, G., T. Lloyd-Braga, and P. Pintus (1998): "Multiple Steady States and Endogenous Fluctuations with Increasing Returns to Scale in Production," Journal of Economic Theory, 80, 60-107.

Cazzavillan, G., and P. Pintus (2004): "Robustness of Multiple Equilibria in OLG Economies," Review of Economic Dynamics, 7, 456-475.

Dixit, A., And J. Stiglitz (1977): "Monopolistic Competition and Optimum Product Diversity," American Economic Review, 67, 297-308.

Dos Santos Ferreira, R., And T. Lloyd-Braga (2005): "Nonlinear Endogenous Fluctuations with Free Entry and Variable Markups," Journal of Economic Dynamics and Control, 29, 849-871.

Duffy, J., And C. PApageorgiou (2000): "A Cross-country Empirical Investigation of the Aggregate Production Function Specification," Journal of Economic Growth, 5, 87-120. 
GALI, J. (1995): "Product Diversity, Endogenous Markups, and Development Traps," Journal of Monetary Economics, 36, 39-63.

Grandmont, J.-M., P. Pintus, and R. De Vilder (1998): "Capitallabour Substitution and Competitive Nonlinear Endogenous Business Cycles," Journal of Economic Theory, 80, 14-59.

Hansen, G. (1985): "Indivisible Labor and the Business Cycle," Journal of Monetary Economics, 16, 309-325.

KunRY, Y. (2001): "Endogenous Fluctuations in a Cournotian Monopolistic Competition Model with Free Entry and Market Power Variability," Research in Economics, 55, 389-412.

Lloyd-Braga, T., C. Nourry, and A. Venditti (2007): "Indeterminacy in Dynamic Models: when Diamond Meets Ramsey," Journal of Economic Theory, 134, 513-536.

Martins, J. O., And S. Scarpetta (1999): "The Levels and Cyclical Behaviour of Mark-ups across Countries and Market Structures," Economics Department Working Papers 213 OCDE.

Nourry, C., And A. VenditTi (2006): "The OLG Model with Endogenous Labor Supply: a General Formulation," Journal of Optimization Theory and Applications, 128, 355-377.

Portier, F. (1995): "Business Formation and Cyclical Markups in the French Business Cycle," Annales d'Economie et de Statistique, 37/38, 411440.

Reichlin, P. (1986): "Equilibrium Cycles in an Overlapping Generations Economy with Production," Journal of Economic Theory, 40, 89-103.

Rotemberg, J. J., And M. Woodford (1991): "Markups and the Business Cycle," in NBER Macroeconomics Annual, ed. by O. J. Blanchard, and S. Fischer, pp. 63-128, Cambridge, Ma. MIT Press.

Seegmuller, T. (2003): "Concurrence Imparfaite, Variabilité du Taux de Marge et Fluctuations Endogènes," Recherches Economiques de Louvain, 69, 371-386.

Yang, X., And B. J. Hejdra (1993): "Monopolistic Competition and Optimum Product Diversity: Comment," American Economic Review, 83, 295-301. 